\title{
Study of prevalence and determinants of polycystic ovarian syndrome among adolescent girls in rural area: a prospective study
}

\author{
Manisha M. Laddad*, Nitin S. Kshirsagar, Gauri P. Shinde, Vaishnavi S. Shivade
}

Department of Obstetrics and Gynecology, Krishna Institute of Medical Sciences, Deemed University, Karad, Maharashtra, India

Received: 20 May 2019

Accepted: 01 July 2019

*Correspondence:

Dr. Manisha M. Laddad,

E-mail: drmanishald@gmail.com

Copyright: (c) the author(s), publisher and licensee Medip Academy. This is an open-access article distributed under the terms of the Creative Commons Attribution Non-Commercial License, which permits unrestricted non-commercial use, distribution, and reproduction in any medium, provided the original work is properly cited.

\begin{abstract}
Background: Polycystic ovarian syndrome (PCOS) is the most frequent endocrinological disorder affecting 5-10\% of women in the reproductive age. This prevalence ranging from $2.2 \%$ to $26 \%$ in adult women from $18-45$ year. In a recent study the prevalence of a confirmed diagnosis of PCOS in adolescents aged 10 to 19 years was 5-15\%, which increased to $10-22 \%$. When undiagnosed cases with documented symptoms qualifying for PCOS according to NIH (National institute of Health) criteria were included. PCOS is a complex endocrine disorder which is most common in women of reproductive age. PCOS may first present in adolescence, but the incidence of PCOS in adolescence is not known, as diagnostic criteria for PCOS in the adolescent age-group is still not defined, PCOS symptoms tend to overlap with normal pubertal changes making the diagnosis even more challenging. The objective is to study prevalence and symptomatology of polycystic ovary syndrome (PCOS) in adolescent girls.

Methods: Prospective Cross sectional study between April 2018 and March 2019. 150 adolescent girls aged 10 to 19 years attending OPD with oligomenorrhea, irregular menstrual cycle, obesity and/or hirsutism were advised for biochemical, hormonal, and ultrasonographic evaluation for diagnosis of PCOS on the basis of Rotterdam's criteria at the Department of Obstetrics and Gynaecology, Krishna Institute of Medical Sciences, Deemed University, Karad, Maharashtra, India.

Results: Prevalence of PCOS in the study was $17.33 \%$ in the study group.

Conclusions: PCOS is increasingly encountered during adolescence, although the overall prevalence is low and evaluation of PCOS in adolescents is challenging. At this age, lifestyle modification is imperative to prevent longterm metabolic and reproductive complications.
\end{abstract}

Keywords: Adolescence, B.M.I, Oligomenorrhea, Hirsutism, PCOS, Waist-Hip ratio

\section{INTRODUCTION}

PCOS is a complex endocrine disorder which is most common in women of reproductive age. ${ }^{1}$ The primary underlying defect in PCOS remains unknown, but key features include insulin resistance, impaired gonadotropin dynamics, and androgen excess. ${ }^{1,2}$ Polycystic ovarian syndrome (PCOS) is the most frequent endocrinological disorder affecting $5-10 \%$ of women in the reproductive age. ${ }^{1}$ This prevalence ranging from $2.2 \%$ to $26 \%$ in adult women from $18-45$ year. ${ }^{2}$ In a recent study the prevalence of a confirmed diagnosis of PCOS in adolescents aged 10 to 19 years was $5-10 \%$, which increased to $10-22 \%$ when undiagnosed cases with documented symptoms qualifying for PCOS according to NIH (National institute of Health) criteria were included. ${ }^{1,2}$ PCOS may first 
present in adolescence, but the incidence of PCOS in adolescence is not known, as diagnostic criteria for PCOS in the adolescent age-group are still not defined. Although polycystic ovarian morphology and features of hyperandrogenism are key factors for the diagnosis of PCOS in adults but adolescent girls during the early stages of puberty tend to have anovulatory menstrual cycles, higher androgen levels, and polycystic ovaries. Thus, PCOS symptoms tend to overlap with normal pubertal changes. ${ }^{3}$ Due to theses variations, the practice of using adult diagnostic criteria raises the concern for misdiagnosis in adolescent age group. ${ }^{4}$ At the same time the diagnosis is important as behavioral modification and lifestyle changes in adolescent age group plays an important role for the prevention of future complications and morbidity. ${ }^{5}$

\section{Why need of study (novelty)}

Because of the heterogeneous nature of the disorder, recognizing adolescents with PCOS may be challenging. However, early recognition and management is important to prevent some of the long-term reproductive and metabolic complications associated with this syndrome. Clinical and USG Criteria are gold standard for diagnosis of PCOS but doing assessment of PCOS by using Fasting insulin, fasting sugar, Free testosterone/LH/FSH is an additional novelty in my study which will be help to improves outcome of my study.

To study prevalence of PCOS and its various clinical aspects in them to determine the gravity of the problem among the adolescent girls 10-19 years in rural areas of Karad attending the adolescents OPD of a tertiary care centre, Krishna Institute of Medical Sciences, Deemed University, Karad, Maharashtra, India.

\section{METHODS}

It's a Cross-sectional study between April 2018 and March 2019. 150 adolescent girls aged 10 to 19 years attending OPD with oligomenorrhea, irregular menstrual cycle, obesity and/or hirsutism were advised for biochemical, hormonal, and ultrasonographic evaluation for diagnosis of PCOS on the basis of Rotterdam's criteria. This study was done at the Department of Obstetrics and Gynaecology; Krishna Institute of Medical Sciences, Deemed University, Karad, Maharashtra, India.

\section{PCOS was defined by Rotterdam's criteria having presence of any two of the three features}

- Oligo/amenorrhea: Absence of menstruation for 45 days or more and/or less than 8 menses per year

- Clinical hyperandrogenism: Modified Ferriman and Gall way (mFG) score of 8 or higher

- Polycystic ovaries: Presence of more than 12 follicles, 2-9 $\mathrm{mm}$ in diameter arranged peripherally, usually combined with increased ovarian volume of more than $10 \mathrm{~cm}^{3}$, and an echo-dense stroma in pelvic ultrasound scan.

\section{Inclusion criteria}

- $\quad$ Adolescent girls age group. (10-19years)

- Adolescent girls had attained menarche

- Adolescents girls who have come to seek treatment from obstetrics and gynaecology and adolescent OPD of Krishna hospital.

\section{Exclusion criteria}

- Those who were known case of thyroid disorders, hyperprolactinemia, Cushing's syndrome, and who were not willing to participate, married and pregnant adolescents were excluded from this study.

A structured questionnaire was given to all the participants. Questionnaire included the following components-knowledge assessment, anthropometric assessment, clinical history; menstrual history included irregularity as well as presence of oligomenorrhea after one year of menarche, wait gain and hirsutism/ androgen production assessment (skin problems, and hair distribution).

Evidence of ovulatory dysfunction included consecutive menstrual intervals of more than 90 days, 1 year after menstrual onset; menstrual intervals persistently less than 21 days or more than 45 days 2 or more years after menarche. Evidence of androgen excess included moderate to severe hirsutism; persistent acne unresponsive to topical therapy; and persistent elevation of serum total and/or free testosterone. Body mass index of up to 23 was taken as normal, between 23 to $24.9 \mathrm{~kg} / \mathrm{m}^{2}$ was taken as overweight, and more than $25 \mathrm{~kg} / \mathrm{m}^{2}$ was considered as obese according to the WHO criteria.

Blood samples were collected between 08:00 and 10:00 hours with an overnight fasting for following blood investigation estimation.

- Fasting BSL and fasting insulin for detection of insulin resistant

- $\quad$ T3, T4 and TSH to rule out hypothyroidism

- $\quad$ LH; FSH; Free Testosterone.

The study was approved by the institutional ethics committee. Written informed consent was taken. All the data was entered in MS excel for statistical analysis.

\section{RESULTS}

Out of 150 adolescent girls enrolled in my study, it was observed that 26 girls had PCOS (According to by Rotterdams criteria). Thus, the prevalence of PCOS in my study was $17.33 \%$. 
Table 1: Distribution according to age group.

\begin{tabular}{|lll|}
\hline Age group & No. & Percentage \\
\hline Early adolescence (10-14) & 7 & $26.92 \%$ \\
\hline Late adolescence (15-19) & 19 & $73.07 \%$ \\
\hline Total & $\mathbf{2 6}$ & $\mathbf{1 0 0}$ \\
\hline
\end{tabular}

In Table 1, the study group was further subdivided into early adolescents means age group 10-14 year and late adolescents means age group 15-19 adolescence; it was observed that PCOS was more prevalent in late adolescence. Age wise distribution of study population shows that the majority, that is $73.07 \%$ of adolescents were in their late adolescence.
Table 2: Distribution according to family history.

\begin{tabular}{|l|l|l|}
\hline Family history & Number & Percentage \\
\hline Yes & 10 & $38.46 \%$ \\
\hline No & 16 & $61.54 \%$ \\
\hline
\end{tabular}

In Table 2 in my study out of 26 PCOS adolescents after taking family history of PCOS it was observed that about $38.46 \%$ had positive family history of PCOS in first degree relative. Four of them gives history of their mother had PCOS in her reproductive life and remaining six gives family history of PCOS in there elder sisters since their adolescent age group. Remaining 16 PCOS means $61.54 \%$ adolescents gives no family history of PCOS in first degree relatives.

Table 3: Association between PCOS and BMI.

\begin{tabular}{|lllll|}
\hline Variables & & PCOS present & PCOS absent & $\%$ \\
\hline \multirow{3}{*}{ BMI } & Non-obese & 11 & $94(75.80 \%)$ & 42.30 \\
\cline { 2 - 5 } & Overweight & 7 & $28(22.58 \%)$ & 26.92 \\
\cline { 2 - 5 } & Obese & 8 & $2(1.61)$ & 30.76 \\
\hline & Total & $\mathbf{2 6}$ & $\mathbf{1 2 4}$ & \\
\hline $\begin{array}{l}\text { Waist/ } \\
\text { hip ratio }\end{array}$ & $<0.85$ & 16 & $116(93.54 \%)$ & 61.53 \\
\cline { 2 - 5 } & $>0.85$ & 10 & $8(6.45 \%)$ & 38.46 \\
\hline
\end{tabular}

In Table 3, among those diagnosed with PCOS, $42.30 \%$ were non-obese, $26.92 \%$ cases were overweight, and $30.76 \%$ were obese. PCOS cases had higher waist to hip ratio and significantly higher BMI.

Table 4: Distribution of cases with menstrual irregularity.

\begin{tabular}{|lll|}
\hline Menstrual irregularity & Number & Percentage \\
\hline PCOS & 22 & 84.61 \\
\hline Non PCOS & 18 & 14.51 \\
\hline
\end{tabular}

Table 5: Other clinical manifestations.

\begin{tabular}{|lll|}
\hline Clinical manifestation & Number & Percentage \\
\hline Acne/oily skin & 16 & 61.53 \\
\hline Hirsutism & 5 & 19.23 \\
\hline Loss of hair/Alopecia & 2 & 7.69 \\
\hline Pigmentation & 8 & 30.76 \\
\hline Mood changes/depression & 4 & 15.38 \\
\hline
\end{tabular}

Menstrual irregularity was the most common presentation in the PCOS group. It was observed in my study total 22 PCOS i.e. $84.61 \%$ cases had abnormal menstrual problems in the form of oligomenorrhoea, amenorrhoea and menorrhagia in Table 4. It was also observed that 18 non PCOS cases i.e.14.51\% had menstrual complaints may be related to puberty changes, hormonal imbalance and other endocrine abnormality responsible for it other than PCOS.
In Table 5 it was observed that Acne or oily skin suggestive of androgenic activity was observed in 61.53 $\%$ of adolescent girls. Hirsutism was found in $19.23 \%$ of cases. Loss of hair was in $7.69 \%$ of girls while pigmentation was in $30.76 \%$ of girls. Mood changes included irritability and depression which was found in $15.38 \%$ girls. Many girls had a combination of symptoms present. Most common being menstrual irregularities and acne.

\section{DISCUSSION}

In adolescents, the exact prevalence of PCOS in India is unknown due to paucity of data. Different studies in India on PCOS have reported prevalence of $2.7 \%$ to $25.5 \%$ and even up to $36 \%$ in adolescents. ${ }^{5}$ The significant variation in different studies is due to lack of consensus on diagnostic criteria.

Prevalence of PCOS in this study was found to be $17.33 \%$, while in the study done by Nidhi et al, the prevalence rate was $14.13 \% .5$ The prevalence was slightly higher than the study done by Nidhi et al which was a community-based study, while the present study was hospital-based study.

When the study group was further subdivided into early and late adolescence it was observed that PCOS was more prevalent in late adolescence. Age wise distribution of study population shows that majority that is $73.07 \%$ 
were in their late adolescence. In similar study done by Dr. Kalavathi et al, and PCOS was more common in late adolescence. $^{6}$ In that study it was observed that about $76.2 \%$ the cases were in late adolescence.

When family history of PCOS was taken it was observed that about $38.46 \%$ had positive family history in first degree relative. In the study done by Kahsar-Miller MD, Nixon C, Boots LR, Go RC, Azziz R, of the 78 mothers and 50 sisters evaluated clinically, 19 (24\%) and 16 $(32 \%)$ were affected with PCOS. ${ }^{7}$ This shows that there is genetic predisposition for PCOS.

Among those diagnosed with PCOS in rural areas, $42.30 \%$ were non-obese, $26.92 \%$ cases were overweight, and $30.76 \%$ were obese. PCOS cases had higher waist to hip ratio and higher BMI. BMI was significantly higher in cases confirmed with PCOS in the study done by Joseph $\mathrm{N}$ et al at Karnataka. ${ }^{8}$ In a study done by Joshi B et al in Mumbai, among those diagnosed with PCOS, $71.8 \%$ were nonobese, $7.5 \%$ cases were overweight, and $20.7 \%$ were obese. ${ }^{9}$

Menstrual irregularity was the most common presentation in the PCOS group. It was observed in $84.61 \%$ cases including oligomenorrhoea, menorrhagia and amenorrhoea. A recent study found that PCOS was the most common underlying etiology in adolescents hospitalized with abnormal uterine bleeding (AUB) and menorrhagia, accounting for $33 \%$ of admissions Maslyanskaya S, Talib HJ, Northridge JL, et al. ${ }^{10}$

Acne or oily skin suggestive of androgenic activity was observed in $61.53 \%$ of adolescent girls. Hirsutism was found in $19.23 \%$ of cases. Loss of hair was in $7.69 \%$ of girls while pigmentation was in $30.76 \%$ of girls. Mood changes included irritability and depression which was found in $15.38 \%$ girls. In Singh A et al, study incidence of mood changes was $14 \% .{ }^{11}$ Many girls had a combination of symptoms present, same results seen in study done by Pathak $G$ et al. ${ }^{12}$ Most common being menstrual irregularities and acne or oily skin suggestive of androgenic activity.

\section{CONCLUSION}

Diagnosis of PCOS in adolescence remains a challenge because of overlapping of symptoms of PCOS with normal pubertal changes in adolescents. Although overall incidence of PCOS in adolescents is low but the incidence of PCOS among adolescence has seen an increasing trend. Early diagnosis is important to inculcate early lifestyle modifications which will prevent metabolic and reproductive complications. Lifestyle modifications for weight reduction and dietary modifications and psychological counselling plays important role in these young girls for preventing long term complications.

\section{ACKNOWLEDGMENTS}

Authors would like to thank adolescents who participated in this study, all colleagues, all postgraduate students in the Department of Obstetrics and Gynecology who cooperated in making the study and the management for encouragement. Special thanks to H.O.D. of Obstetrics and Gynecology, department, and Medical director for supporting and guiding.

\section{Funding: No funding sources}

Conflict of interest: None declared

Ethical approval: The study was approved by the Institutional Ethics Committee

\section{REFERENCES}

1. Azziz R, Carmina E, Dewailly D, DiamantiKandarakis E, Escobar-Morreale HF, Futterweit W, et al. Positions statement: criteria for defining polycystic ovary syndrome as a predominantly hyperandrogenic syndrome: An Androgen Excess Society guideline. J Clin Endocrinol Metab. 2006;91:4237-45.

2. Knochenhauer ES, Key TJ, Kahsar-Miller M, Waggoner W, Boots LR, Azziz R. Prevalence of the polycystic ovary syndrome in unselected black and white women of the southeastern United States: A prospective study. J Clin Endocrinol Metab. 1998;83:3078-82.

3. Kansra AR. Polycystic ovary syndrome in adolescents. J Clinic Outcomes Management. 2016;23(5).

4. Kamboj MK, Bonny AE. Polycystic ovary syndrome in adolescence: diagnostic and therapeutic trategies. Translational Pediatr. 2017;6(4):248.

5. Nidhi R, Padmalatha V, Nagarathna R, Amritanshu $\mathrm{R}$. Prevalence of polycystic ovarian syndrome in Indian adolescents. J Pediatr Adolescent Gynecol. 2011;24(4):223-7.

6. Biradar KD, Shamanewadi AN. A descriptive study of polycystic ovarian syndrome in adolescent girls among a tertiary care hospital of Bangalore. Indian $\mathbf{J}$ Basic Applied Med Res. 2015;4(2):453-5.

7. Kahsar-Miller MD, Nixon C, Boots LR, Go RC, Azziz R. Prevalence of polycystic ovary syndrome (PCOS) in first-degree relatives of patients with PCOS. Fertil Steril. 2001;75(1):53-8.

8. Joseph N, Reddy AG, Joy D, Patel V, Santhosh P, Das S, et al. Study on the proportion and determinants of polycystic ovarian syndrome among health sciences students in South India. J Nat Sci Biol Med. 2016;7(2):166.

9. Joshi B, Mukherjee S, Patil A, Purandare A, Chauhan S, Vaidya R. A cross-sectional study of polycystic ovarian syndrome among adolescent and young girls in Mumbai, India. Indian J Endocrinol Metabol. 2014;18(3):317.

10. Maslyanskaya S, Talib HJ, Northridge JL. Polycystic ovary syndrome: an under-recognized cause of 
abnormal uterine bleeding in adolescents admitted to a children's hospital. J Pediatr Adolesc Gynecol. 2017;30:349.

11. Singh A, Vijaya K. Prevalence of pcos among adolescent girls: a prospective study. IJRCOG. 2018;7;4375.

12. Pathak G, Nichter M. Pcos in globalizing india:an ecosocialpe respective on an emerging lifestyle disease. Soc Sci Med. 2015;146:21-8.
Cite this article as: Laddad MM, Kshirsagar NS, Shinde GP, Shivade VS. Study of prevalence and determinants of polycystic ovarian syndrome among adolescent girls in rural area: a prospective study. Int J Reprod Contracept Obstet Gynecol. 2019;8:3135-9. 